\title{
Kräuterbücher (3)
}

\section{Vom medizinischen Nachschlagewerk zum botanischen Lehrbuch: Zur Entwicklung der Kräuterbuchliteratur}

\author{
Martin Kluge
}

Zahlreiche Naturwissenschaftler des ausgehenden 19. Jahrhunderts legten sich Bibliotheken mit bibliophilen Werkenan.Soauch derETH-Professor derPharmakognosie Carl Hartwich (1851-1917). Seine über 800 Titel umfassende Fachbibliothek, die sich heute als Dauerleihgabe im Pharmazie-Historischen Museum Basel befindet, beinhaltet eine umfangreiche Sammlung alter Kräuterbücher [1]. Diese sind Nachschlagewerke aus dem 16. bis 18. Jahrhundert, in denen Arzneipflanzen mit Anweisungen zu deren medizinischen Gebrauch aufgeführt sind. Auch wennKräuterbücher auf den ersten Blick eine einheitliche Gruppe darstellen, entpuppen sie sich bei näherem Hinsehen als deutlich divergenter, als man dies für diese Büchergattung annehmen würde. Was macht also ein Kräuterbuch aus, und für wen wurde es damals herausgegeben?

Bereits in der Mainzer Urdruckerei, in der Johannes Gutenberg im 15. Jahrhundert den Buchdruck erfand, wurde von Gutenbergs Nachfolger Peter Schöffer (14251503) das erste gedruckte Kräuterbuch herausgegeben: eine in Latein verfasste Zusammenstellung aus damals in antiken und mittelalterlichen Lehrtraktaten überlieferten Quellen zu Heilkräutern und deren medizinischer Anwendung. Zu einem wahren «Bestseller» wurde das Buch, als es 1485 unter dem Namen «Gart der Gesundheit» (Abb. 1) [2] ins Deutsche übersetzt und mit 435 Holzschnitten ver-

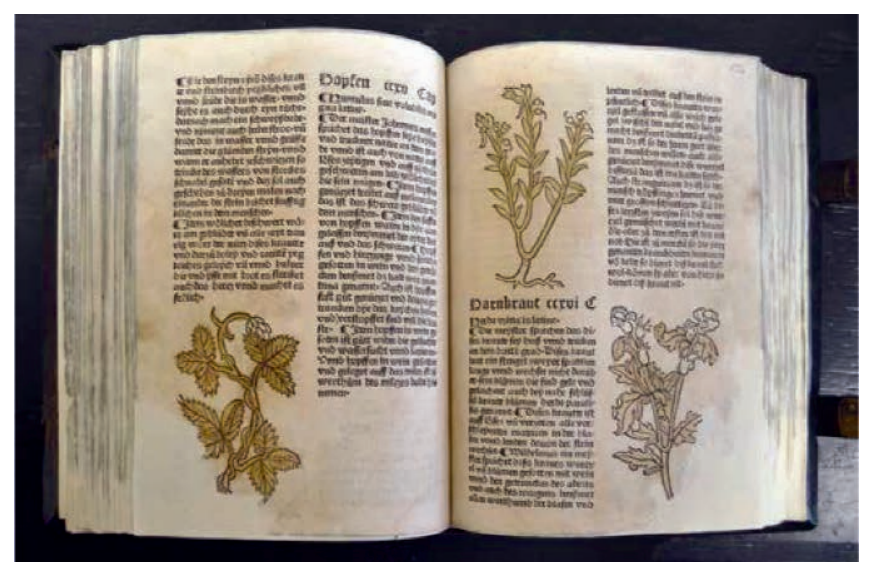

Abb. 1. Johann Wonnecke von Kaub: «Gart der Gesundheit» in der Ausgabe Mainz, 1488. (Foto: Pharmazie-Historisches Museum Basel.) sehen herausgegeben wurde. Etliche Nachdrucke und Neuauflagen liessen nicht lange auf sich warten. Deutschsprachige, reich illustrierte Ausgaben versprachen einen sicheren Absatz, selbst wenn einige von ihnen wissenschaftlich nur wenig Eigenständiges boten. So auch der «Gart der Gesundheit», der mit Erweiterungen von Christian Egenolff, Adam Lonitzer und Peter Uffenbach bis in das späte 18. Jahrhundert immer wieder neu aufgelegt wurde. Warum entsprach das illustrierte deutschsprachige Kräuterbuch derart den Bedürfnissen der damaligen Zeit?

Kräuterbücher richteten sich nicht nur an Apotheker und Ärzte. Diesen standen spätestens seit dem ausgehenden 16. Jahrhundert geeignetere Fachbücher, wie etwa Pharmakopöen, zur Verfügung. In der Folge verschwanden Apotheker daher als Zielpublikum zunehmend aus dem Blickfeld. Vielmehr entsprach die neue Buchgattung den Bedürfnissen einer neuen Käuferschicht: den wohlhabenden, aber oft lateinunkundigen städtischen Patriziern. Für diese erhielt die Abbildung eine Schlüsselrolle. Im Gegensatz zu Apothekern stand ihnen für das Naturstudium in den seltensten Fällen ein artenreicher Kräutergarten zur Verfügung. Möglichst präzise Beschreibungen und naturnahe Illustrationen waren daher für die Identifikation der beschriebenen Heilpflanzen entscheidend.

Auch die Beobachtung, dass Kräuterbücher vor allem eine auf den deutschen Sprachraum beschränkte Buchgattung darstellen, weist darauf hin, dass sie keine direkte Aufgabe mehr in der Apotheke erfüllten. Sie nehmen vielmehr unterschiedliche Überlieferungsstränge der pharmazeutischen Literatur auf und passen sie den jeweiligen Bedürfnissen der Käufer an. Kräuterbücher gehören einerseits zur Ratgeberliteratur für Arzneipflanzen: «Mit den besten Hauss-Artzney-Mitteln», wie es im Titel einer Mattioli-Ausgabe heisst, geben sie medizinischen Laien Ratschläge zur Anwendung von Heilpflanzen. Andererseits gehören Kräuterbücher zur unverzichtbaren Fachliteratur im damals noch jungen Fach Botanik. Und nicht zuletzt zählen sie zur pharmazeutischen Fachliteratur, indem kompendiumartig bestehendes Wissen den jeweiligen Arzneipflanzen zugeordnet wird.
Martin Kluge

Pharmazie-Historisches

Museum der Universität Basel

Totengässlein 3, 4051 Basel, Schweiz

martin.kluge@unibas.ch

www.pharmaziemuseum.ch
Basler Papiermühle

Schweiz. Museum für

Papier, Schrift und Druck

St.-Alban-Tal 37, 4052 Basel, Schweiz

www.papiermuseum.ch 


\section{Mattioli - ein praktizierender Arzt ergänzt antikes Wissen}

Die Vielseitigkeit der Erscheinungsformen von Kräuterbüchern lässt sich exemplarisch am Werk des in Siena geborenen promovierten Arztes Pietro Andrea Mattioli (1501-1577) aufzeigen. Er wirkte als Arzt am Hof des Kardinals in Trient. Von dort aus studierte er, botanisch interessiert, die Flora des südlichen Alpenraums. Gleichzeitig arbeitete er an einer kommentierten Übersetzung von Dioskurides" "De materia medica», der wohl wichtigsten pharmazeutischen Quelle der Antike. Zwischen 1544 und 1551 gab er, mehrfach überarbeitet und jeweils erweitert, seine Kommentarbände heraus. Diese waren anfänglich reine Texteditionen, welchen schliesslich 1554 eine weitere, mit 563 Holzschnitten versehene Ausgabe folgte [3]. Im Stil humanistischer Schriften liess Mattioli den Originaltext des Dioskurides in einer Antiqua-Schrift nach venezianischem Vorbild drucken. Seine eigenen Kommentare erschienen deutlich abgesetzt in Kursiven. 1563, unterdessen als Leibarzt an den Habsburgischen Hof in Prag berufen, gab Mattioli sein Kräuterbuch in deutscher Übersetzung unter dem Namen «Neuw Kreütterbuch» [4] heraus. Im Unterschied zur lateinischen Fassung erschien das Werk nun als grossformatige Prachtausgabe mit nahezu ganzseitigen Holzschnitten, in denen die Pflanzen lebensgross oder sogar in einer darüber hinausgehenden Grösse dargestellt sind (Abb. 2). Der Text der deutschen Ausgabe wurde hingegen unter Verzicht auf das Zitieren antiker Autoritäten deutlich gekürzt. Dies kam den äusserst betuchten Käufern, d.h. reichen Patriziern und Adligen, entgegen, für die das luxuriöse Buch oft weniger Lesestoff als vielmehr ein repräsentatives Schauobjekt darstellte.

Die Nachfrage nach diesem Kräuterbuch riss auch nach dem plötzlichen Pesttod Mattiolis nicht ab. Joachim Camerarius der Jüngere (1534-1598), Arzt und Botaniker in Nürnberg, nutzte die Chance und gab in eigener Be-

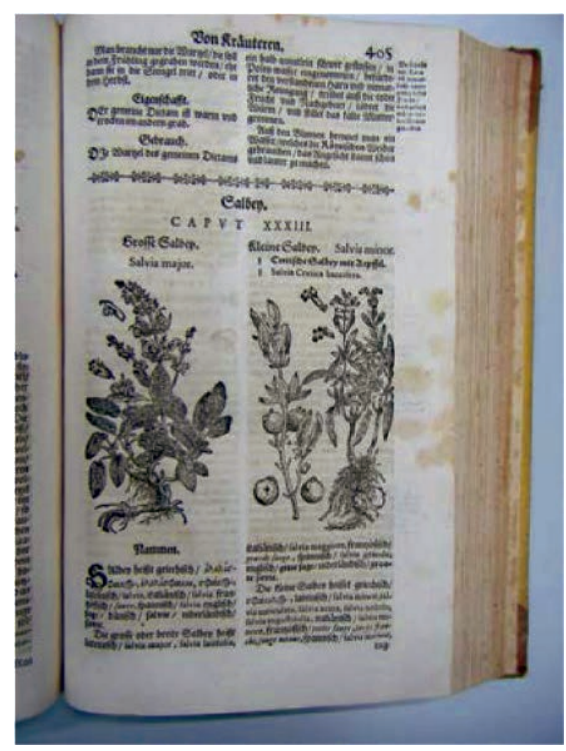

Schweiz Z Ganzheitsmed 2014;26:223-226 arbeitung das Kräuterbuch Mattiolis im Jahr 1586 neu heraus [5]. Auf die kostspieligen Pflanzendarstellungen, die in dieser Grösse und Qualität den Preis für ein Exemplar in gigantische Höhen steigen liessen, verzichtete er und wählte deutlich kleinere Abbildungen, welche er zum Teil aus dem Nachlass Conrad Gessners erwerben konnte. Den Text erweiterte Camerarius zu einem umfangreichen medizinischen Ratgeber - mit umfassenden, nach Beschwerden und Pflanzennamen geordneten Registern. Von der ursprünglichen wissenschaftlichen Auseinandersetzung mit der antiken Vorlage des Dioskurides war in den seither erschienenen deutschen Ausgaben wenig übrig geblieben. Das illustrierte deutschsprachige Kräuterbuch von Mattioli in der Erweiterung von Camerarius wurde dennoch oder gerade deshalb das am weitesten verbreitete Kräuterbücher des 16. und 17. Jahrhunderts.

\section{Otto Brunfels - der Erneuerer}

Bereits eine Generation vor Pietro Andrea Mattioli lässt sich das Aufkommen humanistischen Gedankenguts im Umgang mit Quellen zu Arzneipflanzen erkennen. Als ein Pionier in dieser Arbeit gilt der Universalgelehrte, Theologe und reformatorische Prediger Otto Brunfels (1488-1534). Nachdem er sich in den Wirren der Reformation auf die Seiten der Protestanten geschlagen hatte und freundschaftliche Beziehungen zu Ulrich von Hutten unterhielt, begann er um 1524 als bereits 36-Jähriger mit dem Studium der Naturwissenschaften und der Medizin. Schliesslich promovierte er 1530 in Basel im Fach Medizin. Bereits 2 Jahre später wurde er als Stadtarzt nach Bern berufen, wo er kurz darauf starb. Als Autor trug er in seinem Kräuterbuch (Abb. 3) für jede von ihm aufgeführte Heilpflanze die Beschreibungen der grossen Gelehrten der Antike, wie Dioskurides, Galen, Plinius und weiteren, zusammen. Diesen stellte er eigene Beobachtungen zu einheimischen, in der Natur studierten Pflanzen gegenüber.

Abb. 3.

Otto Brunfels: «Herbarum vivae eicones", Strassburg, 1530. (Foto: PharmazieHistorisches Museum Basel.)

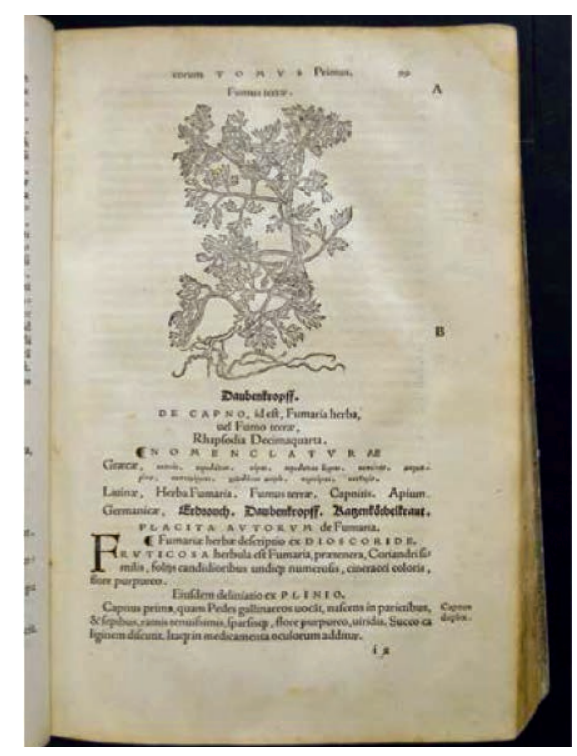


An der medizinischen Kompetenz der antiken Autoritäten zweifelte er nicht: «Dann welche kräuter nit aus Dioscurides/Plinio/Galeno/ und den alten mögen gedeutet werden/ die halt ich alle für ungewissz.» Schwierigkeiten hatte er hingegen damit, ihm bekannte Heilpflanzen den Beschreibungen der Antike zuzuordnen. So diskutiert er beispielsweise, ob es sich beim Rainfarn um die bei Dioskurides beschriebene Ambrosia oder die Athanasia handle. Nur in seltenen Fällen, wenn er für Heilpflanzen bei den antiken Autoritäten keine Entsprechung fand, wie beispielsweise für die Küchenschelle, ergänzte er eigenständig.

Wieder einmal waren für den verlegerischen Erfolg des Buchs die Bilder richtungsweisend. Brunfels liess die Pflanzen, wenn möglich, in ihrer natürlichen Grösse durch den bekannten Strassburger Maler Johann Weiditz zeichnen und in Holz schneiden. Mit der ausserordentlichen Qualität der Holzschnitte, die er direkt von der Pflanze abzeichnen liess, blieb er über die nächsten Jahrhunderte richtungsweisend.

\section{Hieronymus Bock und das botanische Erfassen der Pflanzen}

In der botanischen Auseinandersetzung ging Hieronymus Bock (1498-1554) in seinem Kräuterbuch einen Schritt weiter. Sein Werk ist deutlich umfangreicher und botanisch versierter. Der grosse Erfolg des Werkes beruht auf den sorgsamen Pflanzenbeobachtungen und charakteristischen Beschreibungen, etwa zu den Standorten und Blütezeiten. Sie waren weitaus zutreffender als bei allen bis anhin erschienenen Werken.

Den Errungenschaften der detaillierten Beschreibungen, die Hieronymus Bock als Textautor auszeichnet, stehen Kompromisse bei der Herausgabe des Kräuterbuchs unter anderem in der Bildqualität gegenüber. Bereits in der Systematisierung der Pflanzen unternahm er das nahezu aussichtslose Unterfangen, eigene Beobachtungen mit der überlieferten Pflanzensystematik der Antike in Einklang zu bringen. Wohl im Blickfeld einer nicht ganz so exklusiven, aber dennoch zahlungskräftigen Käuferschicht legte Hieronymus Bock grosses Gewicht auf die Beschreibung der «Kraft und Wirkung» der Heilpflanzen, in die sicher seine langjährige Erfahrung als praktizierender Arzt einfliessen konnte. Auch seine humoristische, wenn nicht gar derbe Sprache verhalf dem Kräuterbuch zu einer breiten Popularität. Dennoch war der Verkaufserfolg des Buchs wesentlich den Zeichnungen des jungen Strassburger Künstlers David Kandel zu verdanken. Die erste, unbebilderte Auflage aus dem Jahr 1539 verkaufte sich nur zögerlich. Erst die zweite Auflage von 1546 kam mit ihren 477 Holzschnitten dem Käuferinteresse entgegen [6]. Die Qualität der Holzschnitte konnte aber mit derjenigen seiner Konkurrenten nicht mithalten. Auffallend oft mussten im Bild die Pflanzen stark verbogen dargestellt werden, um in den gegebenen rechteckigen Bildausschnitt

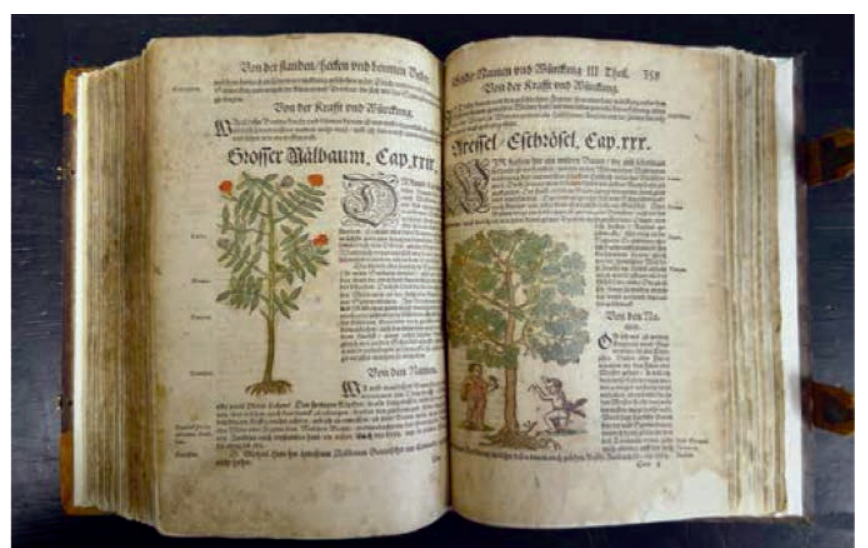

Abb. 4. Hieronymus Bock: Kreüterbuch in der Ausgabe Strassburg, 1987. (Foto: Pharmazie-Historisches Museum Basel.)

zu passen. Gegenstände wie Besen, Biergläser oder Genreszenen wie Bauerntänze, die gelegentlich den Pflanzendarstellungen beigefügt wurden, sind wohl aus verlegerischen Gesichtspunkten eingestreute volkstümliche Komponenten für eine weniger an reiner Wissenschaftlichkeit interessierte Leserschaft (Abb. 4).

\section{Leonhart Fuchs rückt das Bild ins Zentrum}

Die Entstehungsgeschichte des Kräuterbuchs von Leonhart Fuchs (1501-1566) weist viele Parallelen zu den Kräuterbüchern von Otto Brunfels und Mattioli auf. 1542 erschien die lateinische (Abb. 5) und ein Jahr später die deutsche Ausgabe [7, 8]. Wieder unterscheidet sich die lateinische Fassung beträchtlich von der deutschen «Übersetzung». Ähnlich Brunfels trägt Fuchs in der lateinischen Ausgabe im Sinn humanistischer Textkritik zu jeder Pflanze die entsprechenden Passagen der antiken Autoren zusammen. In der deutschen Fassung übersteigt nun die Qualität der Holzschnitte jene der Abbildungen in der lateinischen Ausgabe bei Weitem. Die ganzseitigen Holzschnitte der deutschsprachigen Ausgabe sind Meisterwerke, welche botanisch exakt sowie künstlerisch und technisch hervorragend die gesamte Pflanze mit Wurzel, Stängel, Blättern und Blüten, Samen und Früchten «abgebildet und contrafayt». Oftmals sind bei einer Pflanze die Stadien von der Blüte bis zur reifen Frucht dargestellt. Teilweise wirkt jedoch der Ausdruck der Holzschnitte etwas «blutleer», wenn zugunsten botanischer Details auf räumliche Tiefen durch Schraffierungen verzichtet wurde. Der Verleger ging wahrscheinlich davon aus, dass bei 500 ganzseitigen Tafeln und dem daher enorm hohen Kaufpreis nur sehr betuchte Käufer eine Ausgabe erwerben und es sich leisten können, die Abbildungen nachträglich noch von Hand kolorieren zu lassen.

In der deutschen, vom Bild dominierten Ausgabe wurde der Text ähnlich wie bei Mattioli gekürzt. Die medizinischen Anweisungen unter «Kraft und Wirkung», auf die wohl aus verlegerischen Gesichtspunkten nicht vollkommen verzichtet werden konnten, wurden auf ein 


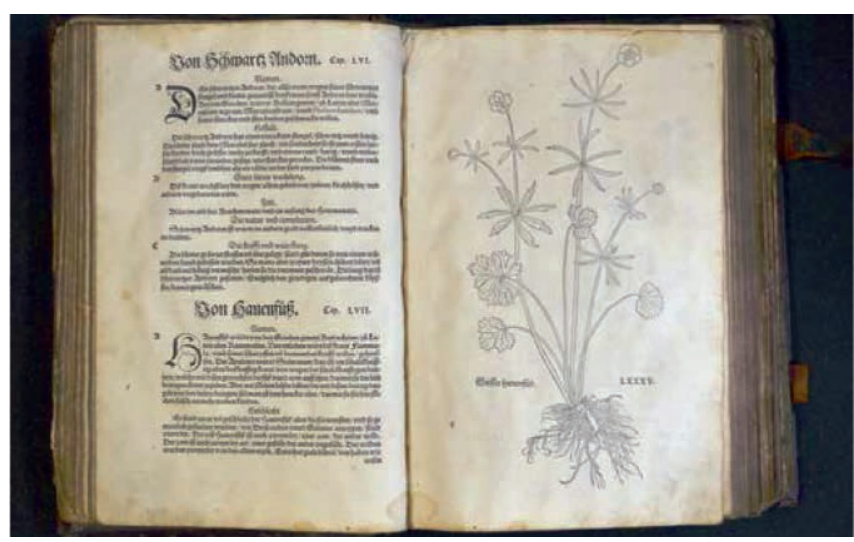

Abb. 5. Leonhart Fuchs: «New Kreüterbuch». Basel, 1542. (Foto: Pharmazie-Historisches Museum Basel.)

Minimum reduziert. Fuchs legt stattdessen Gewicht auf die methodische Beschreibung botanischer Merkmale. Er beschreibt in seinem Kräuterbuch rund 400 Wildgewächse und über 100 Nutz- und Zierpflanzen, für die er in der Natur rund um Tübingen Belege fand. An dem von Fuchs eingeführten System orientierte sich später Carl von Linné.

\section{Quo vadis? Das Kräuterbuch im 17. und 18. Jahrhundert}

Diese «Väter der Botanik», wie sie einst Carl von Linné bezeichnete, zeigen eine Tendenz auf, die sich in den folgenden zwei Jahrhunderten immer deutlicher abzeichnete. Aus den vom Bild dominierten deutschsprachigen grossformatigen Prachtausgaben, bei denen die Funktion als medizinischer Ratgeber zunehmend zurücktrat, entwickelte sich eine in der Landessprache herausgegebene, botanische Fachliteratur. Für diese künstlerisch hochstehende Art der Pflanzendarstellung erwies sich der Holzschnitt zunehmend als zu ungenau und verlangte dem Künstler ausserordentliches Geschick ab. Das Kräuterbuch von Leonhart Fuchs stellt diesbezüglich den Höhe-, aber auch den Endpunkt dar. In Zukunft sollte diese Art der Publikation von kolorierten Kupferstichen abgelöst werden. Christoph Jacob Trew (1695-1769) gab beispielsweise zwischen 1750 und 1773 in einem ambitionierten Projekt unter dem Namen «Plantae selectae» [9] Mappen mit je 10 grossformatigen Kupferstichtafeln her-



Abb. 6. Tabernaemontanus: «Neu-Vollkommen Kräuter-Buch». Basel 1752. (Foto: Departement Chemie und Angewandte Biowissenschaften der ETH Zürich.)

aus. Zeitgleich erschien auch sein in Kupfer gestochenes «Herbarium Blackwellianum» mit einer nach ästhetischen Kriterien getroffenen Bildauswahl, welche die Fremdartigkeit und Schönheit exotischer Pflanzen aufzeigen sollte. Auf diese Editionen wird in einer späteren Folge dieser Reihe näher eingegangen.

Auf der anderen Seite erlebte das Kräuterbuch als medizinischer Ratgeber für Laien weiteren Aufschwung. Bereits Leonhart Fuchs strebte eine auf 1500 Arzneipflanzen erweiterte Ausgabe seines Kräuterbuchs an, die er aber nicht mehr vollenden konnte. Im Laufe des 17. Jahrhunderts stieg der Umfang der neu aufgelegten und ständig erweiterten Kräuterbücher markant an. Den Höhepunkt erreichte Jakob Dietrich aus Bergzabern, der sich Tabernaemontanus (1522-1590) nannte. Seit der Ausgabe von 1687 umfasste sein Kräuterbuch schliesslich mit allen späteren Ergänzungen rund 3000 Pflanzenbeschreibungen mit annähernd 2500 Holzschnitten (Abb. 6) [10]. Es war das umfangreichste seiner Art. Nicht die botanisch exakte Beschreibung einer einheimischen Flora war für diese Nachschlagewerke entscheidend, sondern das Erfassen und Beschreiben möglichst aller Heilpflanzen, inklusive der aus neu entdeckten Kolonien über den Fernhandel importierten Gewächse. Im Laufe des 18. Jahrhunderts, mit neuen wissenschaftlichen Erkenntnissen und veränderten gesellschaftlichen Bedingungen, verschwand schliesslich das Phänomen Kräuterbuch als Buchgattung aus den Verlagsprogrammen genauso abrupt, wie es einst entstand.

\section{Literatur}

1 Brauckmann B: Kräuterbücher (1): Sammelleidenschaft und Forschungseifer: Zwei Gelehrte aus verschiedenen Jahrhunderten auf den Spuren von Opium und Kaffee. Schweiz Z Ganzheitsmed 2014;26:105-107.

2 Gart der Gesundheit. www.botanicus.org/ page/900767.

3 Mattioli PA: Petri Andreae Matthioli com mentarii in libros sex Pedacii Dioscoridis Anazarbei, de medica materia. Digitalisierte Ausgabe: http://digital.ub.uni-duesseldorf.de/ urn/urn:nbn:de:hbz:061:1-160434.
4 Mattioli PA: «Neuw Kreütterbuch». https:// archive.org/details/mobot31753000819281.

5 Mattioli PA: Kreutterbuch deß hochgelehrten unnd weitberühmten Herrn D. Petri Andreae Matthioli. Nachdruck der Ausgabe Frankfurt 1590: http://digital.ub.uni-duesseldorf.de/ vester/content/titleinfo/4025416.

6 Bock H: Kreütterbuch. Digitalisierte Ausgabe von 1595: http://digital.ub.uni-duesseldorf.de/ ihd/content/pageview/4290555.

7 Brauckmann B: Kräuterbücher (2): Publikationskonflikte in der Renaissance: Leonhart
Fuchs und Conrad Gessner im Streit um ihre Pflanzenwerke. Schweiz Z Ganzheitsmed 2014;26:168-171.

8 Fuchs L: New Kreüterbuch. Digitalisiert unter: https://fedora.phaidra.univie.ac.at/ fedora/get/o:58651/bdef:Asset/view.

9 Plantae selectae. http://docnum.u-strasbg.fr/ $\mathrm{cdm} /$ compoundobject/collection/coll13/ id/6063.

10 Theodorus J: Jacobi Theodori Tabernæmontani Neü vollkommen Kräuter-Buch. Basel/ Offenbach am Mäyn, 1731. hbz:061:2-170982. 